\title{
Wick theorem for all orderings of canonical operators
}

\author{
Lajos Diósi \\ Wigner Research Centre for Physics, H-1525 Budapest 114, P. O. Box 49, Hungary
}

\begin{abstract}
Wick's theorem, known for yielding normal ordered from time-ordered bosonic fields, may be generalized for a simple relationship between any two orderings that we define over canonical variables, in a broader sense than before. In this broad class of orderings, the General Wick Theorem (GWT) follows from the Baker-Campbell-Hausdorff identity. We point out that, generally, the characteristic function does not induce an unambigous scheme to order the multiple products of the canonical operators although the value of the ordered product is unique. We construct a manifold of different schemes for each value of $s$ of s-orderings of Cahill and Glauber.
\end{abstract}

\section{INTRODUCTION}

The problem of systematic ordering of canonical operators $\hat{q}, \hat{p}$ appeared first in canonical quantization [1] and also the other way around: in classical phase-space representations of canonically quantized systems [2]. In a different realm, ordering and reordering of quantized fields became central to S-matrix theory [3] 5$]$.

A particular phase-space quasi-distribution $\rho(q, p)$ for a given density matrix $\hat{\rho}$,

$$
\rho(q, p)=\operatorname{tr}[\mathcal{O} \delta(q-\hat{q}) \delta(p-\hat{p}) \hat{\rho}],
$$

requires a particular ordering $\mathcal{O}$ of $\hat{q}$ and $\hat{p}$. The Weyl-Wigner [1, 2], normal [5] and anti-normal orderings are special cases of s-orderings proposed by Cahill and Glauber [6], reviewed together with QP- and PQorderings e.g. in [7].

An ordering $\mathcal{O}$ is traditionally defined by its action on the operator-valued characteristic function. In particular, the Weyl-Wigner ordering $\mathcal{W}$ is defined by this relationship:

$$
\mathcal{W} \mathrm{e}^{a \hat{q}+b \hat{p}}=\mathrm{e}^{a \hat{q}+b \hat{p}}
$$

with the arbitrary c-numbers $a, b$. The normal ordering $\mathcal{N}$ is defined between the annihilation $\hat{c}=(\hat{x}+i \hat{p}) / \sqrt{2}$ and creation $\hat{c}^{\dagger}$ operators (we use units where $\hbar=1$ ):

$$
\mathcal{N} \mathrm{e}^{\lambda^{\star} \hat{c}+\lambda \hat{c}^{\dagger}}=\mathrm{e}^{\lambda \hat{c}^{\dagger}} \mathrm{e}^{\lambda^{\star} \hat{c}},
$$

with an arbitrary complex number $\lambda$. The s-orderings are defined by

$$
\mathcal{O}_{s} \mathrm{e}^{\lambda^{\star} \hat{c}+\lambda \hat{c}^{\dagger}}=\mathrm{e}^{-s|\lambda|^{2} / 2} \mathrm{e}^{\lambda \hat{c}^{\dagger}+\lambda^{\star} \hat{c}}, \quad s \in[-1,1] .
$$

They interpolate between the normal ordering $(s=1)$ and anti-normal ordering $(s=-1)$, with the WeylWigner ordering in the middle $(s=0)$.

Time-ordering $\mathcal{T}$ (also called chronological or pathordering) was proposed first by Dyson [4] in relativistic quantum field theory. The S-matrix is a time-ordered functional of free quantum field operators. If we transform it into the normal ordered form first, the evaluation of matrix elements becomes straightforward. Time- and normal orderings are related by Wick's theorem [5]. For a free relativistic bosonic field $\hat{\varphi}(x)$ it can be written into the compact form, cf. [8]:

$$
\mathcal{T} \mathrm{e} \int J(x) \hat{\varphi}(x) d x=\mathrm{e}^{C} \mathcal{N} \mathrm{e} \int J(x) \hat{\varphi}(x) d x,
$$

where $J(x)$ is an arbitrary c-number field. The exponent in the pre-factor is a c-number:

$$
C=\iint J(x) C(x, y) J(y) d x d y,
$$

where $C(x, y)$ is called the kernel of chronological contraction (or pairing):

$C(x, y)=\mathcal{T} \hat{\varphi}(x) \hat{\varphi}(y)-\mathcal{N} \hat{\varphi}(x) \hat{\varphi}(y) \equiv(\mathcal{T}-\mathcal{N}) \hat{\varphi}(x) \hat{\varphi}(y)$.

The above form of Wick's theorem suggests that a generalized theorem of the same structure holds for any pair $\mathcal{O}$ and $\mathcal{O}^{\prime}$ of orderings.

In Sec. [I] we define the notion of monomial and nonmonomial orderings and we note that, in the latter class, the ordered characteristic function allows for ambiguous schemes of ordering. Sec. III suggests the general Wick theorem for monomial orderings, with a tentative proof in Sec. IV] Sec. Villustrates the theorem on simple applications in quantum mechanics and optics. Sec. V1 proves that an s-ordering of Cahill and Glauber is equivalent with a family of 'redundant' monomial path orderings, and we construct the corresponding family of ordering schemes.

\section{MONOMIAL AND NON-MONOMIAL ORDERINGS}

Let us start with a collection $\left\{\hat{A}_{\alpha}, \alpha \in \Omega\right\}$ of operators to be ordered, where $\Omega$ is a (partially) ordered set of labels. (If $\left[\hat{A}_{\alpha}, \hat{A}_{\beta}\right]=0$ for a certain pair $\alpha, \beta \in \Omega$ then the order of $\alpha$ and $\beta$ may be left unspecified.) Consider an ordered subset $\left\{\alpha_{1}, \alpha_{2}, \ldots \alpha_{n}\right\}=\Omega^{\prime} \subseteq \Omega$ of labels and define the corresponding ordered operator product:

$$
\begin{aligned}
\mathcal{O} \prod_{\alpha \in \Omega^{\prime}} \hat{A}_{\alpha} & =\hat{A}_{\alpha_{n}} \ldots \hat{A}_{\alpha_{3}} \hat{A}_{\alpha_{2}} \hat{A}_{\alpha_{1}} \\
& \equiv\left[\prod_{\alpha \in \Omega^{\prime}} \hat{A}_{\alpha}\right]_{P}
\end{aligned}
$$


where $\mathcal{O}$ stands for the given operator ordering to adopt the ordering $\alpha_{n} \succ \ldots \succ \alpha_{3} \succ \alpha_{2} \succ \alpha_{1}$ of the labels; $[\ldots]_{P}$ is alternative notation referring to the corresponding permutation $P$. The collection of the operators may be redundant: a certain $\hat{A}_{\alpha}$ may coincide with a certain $\hat{A}_{\beta}$ even for $\alpha \neq \beta$. Let us call (8) monomial ordering (or permutation). Ordering, in general, can be the weighted mixture of different monomial orderings:

$$
\mathcal{O} \prod_{\alpha \in \Omega^{\prime}} \hat{A}_{\alpha}=\sum_{P} w_{P}\left[\prod_{\alpha \in \Omega^{\prime}} \hat{A}_{\alpha}\right]_{P}
$$

where $w_{P}$ are non-negative weights that sum up to 1 .

Alternatively, orderings used to be defined by their exponential characteristic functions

$$
\hat{F}_{\mathcal{O}}(\lambda)=\mathcal{O} \prod_{\alpha \in \Omega} \mathrm{e}^{\lambda_{\alpha} \hat{A}_{\alpha}}
$$

at arbitrary arguments $\lambda_{\alpha}$. For monomial orderings (8) they factorize:

$$
\hat{F}_{\mathcal{O}}(\lambda)=\ldots \exp \left(\lambda_{\alpha_{3}} \hat{A}_{\alpha_{3}}\right) \exp \left(\lambda_{\alpha_{2}} \hat{A}_{\alpha_{2}}\right) \exp \left(\lambda_{\alpha_{1}} \hat{A}_{\alpha_{1}}\right)
$$

where $\ldots \succ \alpha_{3} \succ \alpha_{2} \succ \alpha_{1}$ holds. As we see, the value of the characteristic function determines the scheme (8) of monomial ordering (and vice versa). This is not so for non-monomial orderings. The characteristic function does not determine the scheme (9) of ordering uniquely.

Typical non-monomial ordering is the Weyl-Wigner ordering $\mathcal{W}$ defined by its characteristic function (2). It turns a monomial into weighted sum of ordered monomials. For instance:

$$
\mathcal{W} \hat{q}^{2} \hat{p}=\frac{\hat{q}^{2} \hat{p}+\hat{p} \hat{q}^{2}+\hat{q} \hat{p} \hat{q}}{3}=\frac{\hat{q}^{2} \hat{p}+\hat{p} \hat{q}^{2}+2 \hat{q} \hat{p} \hat{q}}{4}
$$

where we show two (of the many) schemes of weighted permutations (9) which correspond to the same nonmonomial ordering $\mathcal{W}$. Still, we anticipate a trick (from Sec. (VI) to show that the Weyl-Wigner ordering can be squeezed into the class of monomial orderings. The trick is that we introduce the redundant collection $\left\{\hat{q}_{\tau}, \hat{p}_{\tau} ; \tau \in\right.$ $[0,1]\}$ of the canonical operators, where $\hat{q}_{\tau}=\hat{q}$ and $\hat{p}_{\tau}=\hat{p}$ for all $\tau \in[0,1]$., i.e.: we assign the time-label $\tau$ formally. Then time-ordering, which is typical monomial ordering, leads to Weyl-Wigner ordering:

$$
\begin{aligned}
\mathcal{T} \exp \left(\int_{0}^{1}\left(a \hat{q}_{\tau}+b \hat{p}_{\tau}\right) d \tau\right) & =\lim _{\epsilon \rightarrow+0}\left(\mathrm{e}^{\epsilon(a \hat{q}+b \hat{p})}\right)^{[1 / \epsilon]}=\mathrm{e}^{a \hat{q}+b \hat{p}}= \\
& =\mathcal{W} \mathrm{e}^{a \hat{q}+b \hat{p}}
\end{aligned}
$$

This is simplest redefinition of $\mathcal{W}$ as a particular monomial time-ordering, there are infinite many other choices which we shall detail and extend for all s-orderings in Sec. VI

We state our general Wick's theorem (Sec. III) for monomial orderings of canonical operators, and construct a tentative proof in Sec. IV. The theorem can not be extended for non-monomial orderings in general. There is, however, a backdoor for some of them, like the above example of Weyl-Wigner ordering, and all s-orderings, cf. in Sec. VI

\section{GENERAL WICK THEOREM}

Consider a canonical system of $n$ pairs of canonical variables $\hat{x}_{k}=\hat{q}_{k}$ and $\hat{x}_{n+k}=\hat{p}_{k}$ respectively, where $\left[\hat{q}_{k}, \hat{p}_{l}\right]=i$ for $k, l=1,2, \ldots, n$. We introduce the linear combinations of the canonical variables:

$$
\hat{X}=\sum_{k=1}^{2 n} a_{k} \hat{x}_{k}
$$

with arbitrary coefficients $\left\{a_{k}\right\}$. Consider a collection of linear combinations

$$
\hat{A}_{\alpha}=\sum_{k=1}^{2 n} A_{\alpha k} \hat{x}_{k}, \quad \alpha \in \Omega,
$$

which form a complete (or overcomplete) basis in the linear space $\{\hat{X}\}$. Let them be the operators to be ordered as given in Sec. II] If $\ldots \succ \alpha_{3} \succ \alpha_{2} \succ \alpha_{1}$ holds then

$$
\begin{aligned}
\mathcal{O} \mathrm{e}^{\hat{X}} & =\mathcal{O} \prod_{\alpha \in \Omega} \mathrm{e}^{\lambda_{\alpha} \hat{A}_{\alpha}} \\
& =\ldots \exp \left(\lambda_{\alpha_{3}} \hat{A}_{\alpha_{3}}\right) \exp \left(\lambda_{\alpha_{2}} \hat{A}_{\alpha_{2}}\right) \exp \left(\lambda_{\alpha_{1}} \hat{A}_{\alpha_{1}}\right),
\end{aligned}
$$

where

$$
\hat{X}=\sum_{\alpha \in \Omega} \lambda_{\alpha} \hat{A}_{\alpha}
$$

It is important to anticipate that whenever we write $\mathcal{O} \mathrm{e}^{\hat{X}}$ or $\mathcal{O} \hat{X}^{2}$, we understand the above linear combination in terms of the operators to be ordered. If $\left\{\hat{A}_{\alpha}\right\}$ forms an overcomplete basis, it matters for the ordering that the coefficients $\left\{\lambda_{\alpha}\right\}$ are not unique. In the case of simple redundancy $\hat{A}_{\alpha}=\hat{A}_{\beta}$ at $\alpha \neq \beta$ we can, if we wish to, remove this ambiguity by collapsing the coefficients: $\lambda_{\alpha}=\lambda_{\beta}$.

To prepare our general Wick theorem (GWT), we consider another collection of operators to be ordered another way $\mathcal{O}^{\prime}$ :

$$
\hat{A}_{a}=\sum_{k=1}^{2 n} A_{a k} \hat{x}_{k}, \quad a \in O .
$$

If $\ldots \succ a_{3} \succ a_{2} \succ a_{1}$ holds then

$$
\begin{aligned}
\mathcal{O}^{\prime} \mathrm{e}^{\hat{X}} & =\mathcal{O}^{\prime} \prod_{a \in O} \mathrm{e}^{\lambda_{a} \hat{A}_{a}} \\
& =\ldots \exp \left(\lambda_{a_{3}} \hat{A}_{a_{3}}\right) \exp \left(\lambda_{a_{2}} \hat{A}_{a_{2}}\right) \exp \left(\lambda_{a_{1}} \hat{A}_{a_{1}}\right)
\end{aligned}
$$

where

$$
\hat{X}=\sum_{a \in O} \lambda_{a} \hat{A}_{a}
$$


We propose the following relationship between the two orderings:

$$
\mathcal{O}^{\prime} \mathrm{e}^{\hat{X}}=\mathrm{e}^{C} \mathcal{O} \mathrm{e}^{\hat{X}}
$$

where the pre-factor is a c-number because the exponent is c-number:

$$
C=\frac{1}{2}\left(\mathcal{O}^{\prime}-\mathcal{O}\right) \hat{X}^{2}
$$

We call it the general contraction between $\mathcal{O}^{\prime}$ and $\mathcal{O}$. This is the GWT, our central result.

If we substitute $\mathcal{O}^{\prime}=\mathcal{W}$ and the identity $\mathcal{W} \hat{X}^{2}=\hat{X}^{2}$, we get a simple equivalent form of GWT:

$$
\begin{aligned}
\mathcal{O} \mathrm{e}^{\hat{X}} & =\mathrm{e}^{C} \mathrm{e}^{\hat{X}} \\
C & =\frac{1}{2}\left(\mathcal{O} \hat{X}^{2}-\hat{X}^{2}\right),
\end{aligned}
$$

where $C$ is a c-number, being the contraction between $\mathcal{O}$ and $\mathcal{W}$. To express its detailed structure, we insert the decomposition (17) of $\hat{X}$ :

$$
\begin{aligned}
C & =\frac{1}{2} \sum_{\alpha, \beta} \lambda_{\alpha} \lambda_{\beta}\left(\mathcal{O} \hat{A}_{\alpha} \hat{A}_{\beta}-\frac{1}{2}\left\{\hat{A}_{\alpha}, \hat{A}_{\beta}\right\}\right) \\
& =\frac{1}{2} \sum_{\alpha \succ \beta} \lambda_{\alpha} \lambda_{\beta}\left[\hat{A}_{\alpha}, \hat{A}_{\beta}\right] .
\end{aligned}
$$

To further simplify the result, we use the expansion (15) of $\hat{A}_{\alpha}$, with the new notations $B_{\alpha k}=A_{\alpha, n+k}$ for the coefficients of the momenta, yielding:

$$
C=\frac{i}{2} \sum_{\alpha \succ \beta} \lambda_{\alpha} \lambda_{\beta} \sum_{k=1}^{n}\left(A_{\alpha k} B_{\beta k}-A_{\beta k} B_{\alpha k}\right) .
$$

We note that in the special case when $\mathcal{O}^{\prime}$ and $\mathcal{O}$ are to order the same collection of operators (in two different ways, of course) then GWT (21,22) can be stated for the characteristic functions as well:

$$
\begin{aligned}
\hat{F}_{\mathcal{O}^{\prime}}(\lambda) & =\exp \left(\frac{1}{2} \sum_{\alpha, \beta} C_{\alpha \beta} \lambda_{\alpha} \lambda_{\beta}\right) \hat{F}_{\mathcal{O}}(\lambda) \\
C_{\alpha \beta} & =\frac{\partial^{2}}{\partial \lambda_{\alpha} \partial \lambda_{\beta}}\left[\hat{F}_{\mathcal{O}^{\prime}}(\lambda)-\hat{F}_{\mathcal{O}}(\lambda)\right]_{\lambda=0} .
\end{aligned}
$$

\section{TENTATIVE PROOF}

Let us consider a given $\hat{X}$, as in (14), and its decompositions (17) and (20):

$$
\hat{X}=\sum_{k=1}^{2 n} a_{k} \hat{x}_{k}=\sum_{\alpha \in \Omega} \lambda_{\alpha} \hat{A}_{\alpha}=\sum_{a \in O} \lambda_{a} \hat{A}_{a}
$$

The proof of GWT (21,22) simplifies if we restrict ourselves for the case when all coefficients $a_{k}, A_{\alpha k}, A_{a k}$ are non-negative. Then we construct the asymptotic form of the maximally refined decomposition of $\hat{X}$ :

$$
\hat{X}=\lim _{\epsilon \rightarrow+0} \sum_{k=1}^{2 n}(\underbrace{\epsilon \hat{x}_{k}+\epsilon \hat{x}_{k}+\ldots+\epsilon \hat{x}_{k}}_{\left[a_{k} / \epsilon\right]})
$$

If we approach the limit $\epsilon \rightarrow 0$, the two orderings in question act on the same (yet unordered) product of exponentials:

$$
\begin{aligned}
& \mathcal{O} \mathrm{e}^{\hat{X}}=\lim _{\epsilon \rightarrow+0} \mathcal{O} \\
& \mathcal{O}^{\prime} \mathrm{e}^{\hat{X}}=\lim _{\epsilon \rightarrow+0} \mathcal{O}^{\prime}
\end{aligned}\left\{\prod_{k=1}^{2 n}(\underbrace{\underbrace{\epsilon \hat{x}_{k}} \times \mathrm{e}^{\epsilon \hat{x}_{k}} \times \ldots \times \mathrm{e}^{\epsilon \hat{x}_{k}}}_{\left[a_{k} / \epsilon\right]}),\right.
$$

where the number of factors $\mathrm{e}^{\epsilon \hat{x}_{k}}$ is $\left[a_{k} / \epsilon\right]$ for all $k$. It will be the order of the factors, and nothing else, that distinguishes $\mathcal{O}$ and $\mathcal{O}^{\prime}$. From one to the other, we can go by a finite sequence $P_{1}, P_{2}, \ldots, P_{K}$ of $K$ switches between neighboring factors. ( $K \rightarrow \infty$ for $\epsilon \rightarrow+0$.) Namely,

$$
\mathcal{O}^{\prime} \mathrm{e}^{\hat{X}}=\mathcal{P}_{K} \ldots \mathcal{P}_{2} \mathcal{P}_{1} \mathcal{O} \mathrm{e}^{\hat{X}}
$$

where we understand the same (redundant) factorization (30) for $\mathrm{e}^{\hat{X}}$. Let us consider the r.h.s. of the above equation and suppose $\mathcal{P}_{K}$ inverts the order of neighbors $\mathrm{e}^{\epsilon \hat{x}_{k}} \mathrm{e}^{\epsilon \hat{x}_{l}}$ in $\mathcal{P}_{K-1} \ldots \mathcal{P}_{1} \mathcal{O} \mathrm{e}^{\hat{X}}$ :

$$
\mathcal{P}_{K} \mathrm{e}^{\epsilon \hat{x}_{k}} \mathrm{e}^{\epsilon \hat{x}_{l}}=\mathrm{e}^{\epsilon \hat{x}_{l}} \mathrm{e}^{\epsilon \hat{x}_{k}} \equiv \mathrm{e}^{\epsilon^{2}\left[\hat{x}_{l}, \hat{x}_{k}\right]} \mathrm{e}^{\epsilon \hat{x}_{k}} \mathrm{e}^{\epsilon \hat{x}_{l}},
$$

where we used the simple Baker-Campbell-Hausdorff [9] identity. We can write:

$$
\mathcal{P}_{K} \ldots \mathcal{P}_{1} \mathcal{O} \mathrm{e}^{\hat{X}}=\mathrm{e}^{\epsilon^{2}\left[\hat{x}_{l}, \hat{x}_{k}\right]} \mathcal{P}_{K-1} \ldots \mathcal{P}_{1} \mathcal{O} \mathrm{e}^{\hat{X}}
$$

Observe that

$$
\mathcal{P}_{K} \ldots \mathcal{P}_{1} \mathcal{O} \hat{X}^{2}-\mathcal{P}_{K-1} \ldots \mathcal{P}_{1} \mathcal{O} \hat{X}^{2}=2 \epsilon^{2}\left[\hat{x}_{l}, \hat{x}_{k}\right]
$$

Using this, the r.h.s. of (33) reads:

$$
\mathrm{e}^{\frac{1}{2}\left(\mathcal{P}_{K} \ldots \mathcal{P}_{1}-\mathcal{P}_{K-1} \ldots \mathcal{P}_{1}\right) \mathcal{O} \hat{X}^{2}} \mathcal{P}_{K-1} \ldots \mathcal{P}_{1} \mathcal{O} \mathrm{e}^{\hat{X}}
$$

We repeat the same identity transformation on the factor $\mathcal{P}_{K-1} \ldots \mathcal{P}_{1} \mathcal{O} \mathrm{e}^{\hat{X}}$, yielding

$$
\mathrm{e}^{\frac{1}{2}\left(\mathcal{P}_{K} \ldots \mathcal{P}_{1}-\mathcal{P}_{K-2} \ldots \mathcal{P}_{1}\right) \mathcal{O} \hat{X}^{2}} \mathcal{P}_{K-2} \ldots \mathcal{P}_{1} \mathcal{O} \mathrm{e}^{\hat{X}}
$$

After $K$ steps, we get

$$
\mathrm{e}^{\frac{1}{2}\left(\mathcal{P}_{K} \ldots \mathcal{P}_{1} \mathcal{O}-\mathcal{O}\right) \hat{X}^{2}} \mathcal{O} \mathrm{e}^{\hat{X}}=\mathrm{e}^{\frac{1}{2}\left(\mathcal{O}^{\prime}-\mathcal{O}\right) \hat{X}^{2}} \mathcal{O} \mathrm{e}^{\hat{X}}
$$

where we used $\mathcal{O}^{\prime}=\mathcal{P}_{K} \ldots \mathcal{P}_{1} \mathcal{O}$ from (31). This is our ultimate expression for the r.h.s. of (21). Our GWT is confirmed.

Recall that we restricted the proof: the operators to be ordered had to be linear combinations of the canonical variables with non-negative coefficients. The maximally refined decomposition (29) contained terms with 
the unique positive coefficient $\epsilon$. If we want to extend the proof for orderings of operators without the above restriction, we have to construct the maximally refined decomposition for all $\hat{X}$. There will be four types of coefficients: $\pm \epsilon, \pm i \epsilon$ instead of the positive ones $\epsilon$. Once we have constructed such a maximally refined decomposition to host both $\mathcal{O}$ and $\mathcal{O}^{\prime}$, the proof follows the same steps as before - and needs a definitely more intricate book-keeping of the four types of exponentials. Intuition says, nonetheless, that the validity of our theorem extends from positive parameters for all complex ones just by the theorem's analytic form.

\section{EXAMPLES: $\mathcal{T}$ VERSUS QP VERSUS $\mathcal{N}$ ORDERINGS}

We are going to illustrate the flexibility of GWT in solving simple tasks in elementary quantum mechanics and quantum optics.

Consider the Schrödinger equation $\dot{\psi}_{t}=-\hat{H}_{t} \psi_{t}$ of a mass $m$, where the Hamiltonian $\hat{H}_{t}=\left(\hat{p}^{2} / 2 m\right)-F_{t} \hat{q}$ contains a given time-dependent force. Let us find the solution $\psi_{t}^{I}=\hat{U}_{t} \psi_{0}$ in the interaction picture where $\hat{q}_{t}=$ $\hat{q}+\hat{p} t / m$ and $\hat{p}_{t}=\hat{p}$. (As is known, the solution $\psi_{t}$ in Schrödinger picture is obtained if we solve the force-free Schrödinger equation with $\psi_{t}^{I}$ as the initial state.) The evolution operator in interaction picture is a $\mathcal{T}$-ordered exponential:

$$
\hat{U}_{t}=\mathcal{T} \mathrm{e}^{\hat{X}_{t}}
$$

where $\hat{X}_{t}$ is linear combination of the canonical variables:

$$
\hat{X}_{t}=i \int_{0}^{t} F_{\tau} \hat{q}_{\tau} d \tau
$$

The r.h.s. is the chronological decomposition of $\hat{X}_{t}$ : the summation label $\alpha \in \Omega$ in (17) became the integral variable $\tau \in(0, t)$, the set $\left\{\hat{A}_{\alpha}\right\}$ became $\left\{\hat{q}_{\tau}\right\}$, the coefficients $\lambda_{\alpha}$ became $i F_{\tau}$. To evaluate $\hat{U}_{t}$, we shall consider its QPordering, hence we need a canonical decomposition of $\hat{X}_{t}$ :

$$
\hat{X}_{t}=i \int_{0}^{t} F_{\tau} d \tau \hat{q}+\frac{i}{m} \int_{0}^{t} F_{\tau} \tau d \tau \hat{p} \equiv i \Delta p_{t} \hat{q}-i \Delta q_{t} \hat{p}
$$

where we introduced the momentum and coordinate shifts $\Delta p_{t}, \Delta q_{t}$ for notational convenience. The label $a$ in (20) takes two values only: $\hat{A}_{a=1}=\hat{q}$ and $\hat{A}_{a=2}=\hat{p}$, also $\lambda_{a=1}=i \Delta p_{t}$ and $\lambda_{a=2}=-i \Delta q_{t}$. The QP-ordering $\mathcal{O}_{Q P}$ pushes all $\hat{q}$ to the left of all $\hat{p}$. Now we apply our GWT (21) to (38),

$$
\hat{U}_{t}=\mathcal{T} \mathrm{e}^{\hat{X}_{t}}=\mathrm{e}^{C_{t}} \mathrm{e}^{i \Delta p_{t} \hat{q}} \mathrm{e}^{-i \Delta q_{t} \hat{p}},
$$

with the contraction (22):

$$
C_{t} \equiv \frac{1}{2}\left(\mathcal{T}-\mathcal{O}_{Q P}\right) \hat{X}_{t}^{2}=\frac{i}{2} \int_{0}^{t} \int_{0}^{t} F_{\tau} F_{\sigma}|\tau-\sigma| d \tau d \sigma .
$$

$\hat{U}_{t} \psi_{0}$ provides the following explicit solution for the wave function in the interaction picture:

$$
\psi_{t}^{I}(q)=\mathrm{e}^{C_{t}} \mathrm{e}^{i \Delta p_{t} q} \psi_{0}\left(q-\Delta q_{t}\right) .
$$

A similar standard task is the electromagnetic cavity oscillator of frequency $\omega$ under external driving. The Hamiltonian reads: $\hat{H}_{t}=\omega \hat{c}^{\dagger} \hat{c}+\left(E_{t}^{*} \hat{c}-\right.$ H.c. $)$. In interaction picture, where $\hat{c}_{t}=\mathrm{e}^{-i \omega t} \hat{c}$ and $\hat{c}_{t}^{\dagger}=\mathrm{e}^{i \omega t} \hat{c}^{\dagger}$, the evolution operator is (38) again, with

$$
\hat{X}_{t}=\int_{0}^{t}\left(E_{\tau}^{*} \hat{c}_{\tau}-\text { H.c. }\right) d \tau \equiv \Delta c_{t}^{\star} \hat{c}-\text { H.c. }
$$

We can rewrite the evolution operator $\hat{U}_{t}$ into the normal ordered form, applying the GWT (21, 22):

$$
\begin{gathered}
\hat{U}_{t}=\mathrm{e}^{\frac{1}{2}(\mathcal{T}-\mathcal{N}) \hat{X}_{t}^{2}} \mathcal{N} \mathrm{e}^{\hat{X}_{t}}=\mathrm{e}^{C_{t}} \mathrm{e}^{-\Delta c_{t} \hat{c}^{\dagger}} \mathrm{e}^{\Delta c_{t}^{\star} \hat{c}}, \\
C_{t}=-\int_{0}^{t} \int_{0}^{t} \theta(\tau-\sigma) E_{\tau}^{*} E_{\sigma} \mathrm{e}^{-i \omega(\tau-\sigma)} d \tau d \sigma .
\end{gathered}
$$

In quantum optics, there is a spectacularly simple definition of the squeezing operator with squeezing parameter $\mu>0$ :

$$
\hat{S}=\frac{1}{\sqrt{\mu}} \int|q / \mu\rangle\langle q| d q=\mathcal{O}_{P Q} \mathrm{e}^{i(1-1 / \mu) \hat{p} \hat{q}},
$$

where the first expression was proposed in Refs. 10] while the second compact form is our finding based on it. To achieve the normal ordered form, which was the task in Refs. [10] as well, we first unravel the bilinear form $\hat{p} \hat{q}$ in the exponent. Introduce $\kappa=1-1 / \mu$ and consider

$$
\hat{S}=\mathcal{O}_{P Q} \mathrm{e}^{i \kappa \hat{p} \hat{q}}=\int \mathcal{O}_{P Q} \mathrm{e}^{i z \hat{p} \pm z^{\star} \hat{q}} \exp \left(-\frac{|z|^{2}}{|\kappa|}\right) \frac{d^{2} z}{\pi|\kappa|}
$$

where the $\operatorname{sign} \pm$ is the sign of $\kappa$. Now we can apply our GWT (21, 22):

$$
\mathcal{O}_{P Q} \mathrm{e}^{i z \hat{p} \pm z^{\star} \hat{q}}=\mathrm{e}^{\frac{1}{4}\left(z^{\star}\right)^{2}-\frac{1}{4} z^{2} \pm \frac{1}{2}|z|^{2}} \mathcal{N} \mathrm{e}^{i z \hat{p} \pm z^{\star} \hat{q}} .
$$

Inserting this in (48) and performing the complex integral, we obtain:

$$
\begin{aligned}
\hat{S} & =\sqrt{\frac{2 \mu}{1+\mu^{2}}} \mathcal{N} \exp \left\{\frac{-i\left(1-\mu^{2}\right) \hat{p} \hat{q}-\frac{1}{2}(1-\mu)^{2}\left(\hat{p}^{2}+\hat{q}^{2}\right)}{1+\mu^{2}}\right\}= \\
& =\sqrt{\frac{2 \mu}{1+\mu^{2}}} \mathcal{N} \exp \left\{\frac{-\frac{1}{2}\left(1-\mu^{2}\right)\left(\hat{c}^{2}-\hat{c}^{\dagger}\right)+(1-\mu)^{2} \hat{c}^{\dagger} \hat{c}}{1+\mu^{2}}\right\} .
\end{aligned}
$$

\section{S-ORDERING}

The s-orderings are defined by their characteristic function (44). Apart from the two marginal cases $s= \pm 1$ they are non-monomial and the induced scheme (9) of ordering is not unique. First we redefine a given s-ordering as a path (i.e.: monomial) ordering of a redundant collection of the canonical operators, then we can construct the corresponding scheme of their s-ordering. 


\section{A. s-Ordering as Path Ordering}

We are going to show that an s-ordering is equivalent with path-orderings $\mathcal{T}$ of a (redundant) collection of operators $\left\{\hat{c}_{\tau}, \hat{c}_{\tau}^{\dagger} ; \tau \in[0,1]\right\}$ where we take $\hat{c}_{\tau} \equiv \hat{c}$ and $\hat{c}_{\tau}^{\dagger} \equiv \hat{c}^{\dagger}$ after the path-ordering only. To define a concrete ordering, we must first introduce a decomposition of the exponent $\hat{X}=\lambda^{\star} \hat{c}+\lambda \hat{c}^{\dagger}$. Let us choose the following structure:

$$
\hat{X}=\int_{0}^{1}\left(\lambda^{\star} \hat{c}_{\tau} d \tau+\lambda \hat{c}_{\tau}^{\dagger} d \chi_{\tau}\right)
$$

where $\chi_{0}=0$ and $\chi_{1}=1$, also $\chi_{\tau}$ must be monotonous. As we said, we cancel the label $\tau$ of $\hat{c}, \hat{c}^{\dagger}$ after the pathordering. Along the path, the rate of $\hat{c}^{\dagger}$ 's versus the constant rate of $\hat{c}$ 's is ruled by $\chi_{\tau}$. We prove below that this path-ordering is equivalent with s-ordering (4):

$$
\mathcal{O}_{s} \mathrm{e}^{\lambda^{\star} \hat{c}+\lambda \hat{c}^{\dagger}}=\left.\mathcal{T} \exp \left\{\int_{0}^{1}\left(\lambda^{\star} \hat{c}_{\tau} d \tau+\lambda \hat{c}_{\tau}^{\dagger} d \chi_{\tau}\right)\right\}\right|_{\hat{c}_{\tau}=\hat{c}},
$$

provided $\chi_{\tau}$ satisfies

$$
s=1-2 \int_{0}^{1} \chi_{\tau} d \tau .
$$

The proof goes like this. Considering the decomposition (51) of $\hat{X}$, we write the r.h.s. of (52) as $\mathcal{T} \mathrm{e}^{\hat{X}}$ and apply (23,24) to it:

$$
\mathcal{T} \mathrm{e}^{\hat{X}}=\mathrm{e}^{C} \mathrm{e}^{\lambda^{\star} \hat{c}+\lambda \hat{c}^{\dagger}}
$$

where

$$
C=\frac{1}{2}\left(\mathcal{T} \hat{X}^{2}-\hat{X}^{2}\right)=|\lambda|^{2}\left(\int_{0}^{1} \chi_{\tau} d \tau-\frac{1}{2}\right) .
$$

The steps that led to this expression of $C$ were the following.

$\mathcal{T} \hat{X}^{2}=\int_{0}^{1} \int_{0}^{1} \mathcal{T}\left(\lambda^{\star} \hat{c}_{\tau} d \tau+\lambda \hat{c}_{\tau}^{\dagger} d \chi_{\tau}\right)\left(\lambda^{\star} \hat{c}_{\sigma} d \sigma+\lambda \hat{c}_{\sigma}^{\dagger} d \chi_{\sigma}\right)$.

Let us write $\hat{X}^{2}$ into the similar integral form:

$$
\hat{X}^{2}=\int_{0}^{1} \int_{0}^{1}\left(\lambda^{\star} \hat{c} d \tau+\lambda \hat{c}^{\dagger} d \chi_{\tau}\right)\left(\lambda^{\star} \hat{c} d \sigma+\lambda \hat{c}^{\dagger} d \chi_{\sigma}\right) .
$$

Then we get

$$
\begin{aligned}
& 2 C=\mathcal{T} \hat{X}^{2}-\hat{X}^{2}= \\
= & |\lambda|^{2} \int_{0}^{1} \int_{0}^{1}\left\{\left(\mathcal{T} \hat{c}_{\tau} \hat{c}_{\sigma}^{\dagger}-\hat{c} \hat{c}^{\dagger}\right) d \tau d \chi_{\sigma}+\left(\mathcal{T} \hat{c}_{\tau}^{\dagger} \hat{c}_{\sigma}-\hat{c}^{\dagger} \hat{c}\right) d \chi_{\tau} d \sigma\right\} \\
= & |\lambda|^{2} \int_{0}^{1} \int_{0}^{1} \theta(\sigma-\tau)\left(d \chi_{\tau} d \sigma-d \tau d \chi_{\sigma}\right) \\
= & |\lambda|^{2}\left(2 \int_{0}^{1} \chi_{\sigma} d \sigma-1\right) .
\end{aligned}
$$

Therefore (54), i.e., the r.h.s. of (52), reads:

$$
\mathcal{T} \mathrm{e}^{\hat{X}}=\exp \left(|\lambda|^{2} \int_{0}^{1} \chi_{\tau} d \tau-\frac{|\lambda|^{2}}{2}\right) \mathrm{e}^{\lambda^{\star} \hat{c}+\lambda \hat{c}^{\dagger}} .
$$

This, at the condition (53), becomes $\mathcal{O}_{s} \mathrm{e}^{\lambda^{\star} \hat{c}+\lambda \hat{c}^{\dagger}}$ as defined by (4).

\section{B. Non-monomial schemes induced by s-ordering}

Derivatives of the characteristic function (44) yield the s-ordered products of the canonical operators $\hat{c}, \hat{c}^{\dagger}$ :

$$
\begin{aligned}
\mathcal{O}_{s} \hat{c}^{n}\left(\hat{c}^{\dagger}\right)^{m} & =\left.\left(\frac{\partial}{\partial \lambda^{\star}}\right)^{n}\left(\frac{\partial}{\partial \lambda}\right)^{m} \mathcal{O}_{s} \mathrm{e}^{\lambda^{\star} \hat{c}+\lambda \hat{c}^{\dagger}}\right|_{\lambda=0} \\
& =\left.\left(\frac{\partial}{\partial \lambda^{\star}}\right)^{n}\left(\frac{\partial}{\partial \lambda}\right)^{m} \mathrm{e}^{-s|\lambda|^{2} / 2} \mathrm{e}^{\lambda \hat{c}^{\dagger}+\lambda^{\star}} \hat{c}\right|_{\lambda=0},
\end{aligned}
$$

for $n, m=0,1,2, \ldots$ Beyond the value $\mathcal{O}_{s} \hat{c}^{n}\left(\hat{c}^{\dagger}\right)^{m}$, our point of interest is the scheme (9) of ordering:

$$
\mathcal{O}_{s} \hat{c}^{n}\left(\hat{c}^{\dagger}\right)^{m}=\sum_{P=1}^{(n+m) !} w_{P}\left[\hat{c}^{n}\left(\hat{c}^{\dagger}\right)^{m}\right]_{P}
$$

where $w_{P} \geq 0$ is the weight of the $P$ th permutation, the weights are normalized; $\left[\hat{c}^{n}\left(\hat{c}^{\dagger}\right)^{m}\right]_{P}$ stands for the $P$ th permutation of the factors.

We shall consider $\mathcal{O}_{s} \hat{c}^{n}\left(\hat{c}^{\dagger}\right)^{m}$ for $n=m=1$ first. If we wish to bring it into the form on the r.h.s. of (61), we get the following unique result:

$$
\mathcal{O}_{s} \hat{c} \hat{c}^{\dagger}=\frac{1+s}{2} \hat{c}^{\dagger} \hat{c}+\frac{1-s}{2} \hat{c} \hat{c}^{\dagger} .
$$

As expected, $s \in[-1,1]$ interpolates between normal and anti-normal orderings. Next, let us take $n=2, m=1$. It is easy to derive, e.g., the following two forms (out of an infinite family):

$$
\mathcal{O}_{s} \hat{c}^{2} \hat{c}^{\dagger}=\hat{c}^{\dagger} \hat{c}^{2}+(1-s) \hat{c}=\hat{c}^{2} \hat{c}^{\dagger}-(1+s) \hat{c} .
$$

Using the identity $\left[\hat{c}, \hat{c}^{\dagger}\right]=1$, we could bring these two to two different forms, resp., both of them conform with the r.h.s. of (61). But the issue grows unmanageable for higher powers $n, m$ unless we find a systematic way. Let us use our concept of orderings and the GTW (Sec. III).

Inserting (52) into (60), we obtain the general expression

$$
\begin{aligned}
& \mathcal{O}_{s} \hat{c}^{n}\left(\hat{c}^{\dagger}\right)^{m}=\left.\mathcal{T}\left(\int_{0}^{1} \hat{c}_{\tau} d \tau\right)^{n}\left(\int_{0}^{1} \hat{c}_{\tau}^{\dagger} d \chi_{\tau}\right)^{m}\right|_{\hat{c}_{\tau}=\hat{c}} \\
= & \left.\int_{0}^{1} d \tau_{1} \ldots \int_{0}^{1} d \tau_{n} \int_{0}^{1} d \chi_{\sigma_{1}} \ldots \int_{0}^{1} d \chi_{\sigma_{m}} \mathcal{T} \hat{c}_{\tau_{1}} \ldots \hat{c}_{\tau_{n}} \hat{c}_{\sigma_{1}}^{\dagger} \ldots \hat{c}_{\sigma_{m}}^{\dagger}\right|_{\hat{c}_{\tau}=\hat{c}},
\end{aligned}
$$

which is already conform with the structure on the r.h.s. of (61). Here we work out the special case $n=2, m=1$ 
only:

$$
\begin{aligned}
\mathcal{O}_{s} \hat{c}^{2} \hat{c}^{\dagger} & =\left.\int_{0}^{1} d \chi_{\tau} \int_{0}^{1} d \sigma_{1} \int_{0}^{1} d \sigma_{2} \mathcal{T} \hat{c}_{\tau}^{\dagger} \hat{c}_{\sigma_{1}} \hat{c}_{\sigma_{2}}\right|_{\hat{c}_{\tau}=\hat{c}} \\
& =w_{1} \hat{c}^{\dagger} \hat{c} \hat{c}+w_{2} \hat{c} \hat{c}^{\dagger} \hat{c}+w_{3} \hat{c} \hat{c} \hat{c}^{\dagger},
\end{aligned}
$$

with

$$
\begin{aligned}
w_{1} & =\int_{0}^{1} d \chi_{\tau} \int_{0}^{1} d \sigma_{1} \int_{0}^{1} d \sigma_{2} \theta\left(\tau-\sigma_{1}\right) \theta\left(\tau-\sigma_{2}\right) \\
& =\int_{0}^{1} d \chi_{\tau} \tau^{2} \\
w_{2} & =2 \int_{0}^{1} d \chi_{\tau} \int_{0}^{1} d \sigma_{1} \int_{0}^{1} d \sigma_{2} \theta\left(\sigma_{1}-\tau\right) \theta\left(\tau-\sigma_{2}\right) \\
& =2 \int_{0}^{1} d \tau \int_{0}^{\tau} d \chi_{\tau} \tau \\
w_{3} & =\int_{0}^{1} d \chi_{\tau} \int_{0}^{1} d \sigma_{1} \int_{0}^{1} d \sigma_{2} \theta\left(\sigma_{1}-\tau\right) \theta\left(\sigma_{2}-\tau\right) \\
& =2 \int_{0}^{1} d \tau \int_{0}^{\tau} d \tau \chi_{\tau} .
\end{aligned}
$$

The constraint $w_{1}+w_{2}+w_{3}=1$ is satisfied by construction. The three integrals (66) depend on the choice of $\chi_{\tau}$. If we take a simplest function $\chi_{\tau}=\tau^{\kappa}$ with $\kappa=\frac{1+s}{1-s} \geq 1$, covering the values $s \in[0,1)$, we get

$$
\mathcal{O}_{s} \hat{c}^{\dagger} \hat{c}^{2}=\frac{(1-s) \hat{c}^{\dagger} \hat{c} \hat{c}+\left(1-s^{2}\right) \hat{c} \hat{c}^{\dagger} \hat{c}+(1+s)^{2} \hat{c} \hat{c} \hat{c}^{\dagger}}{3+s} .
$$

Obviously, other functions $\chi_{\tau}$ would have resulted in different weights $w_{1}, w_{2}, w_{3}$, i.e., in different schemes (61) of the same non-monomial s-ordering.

\section{CONCLUDING REMARKS}

In 1950, Wick's theorem between normal and chronological orderings of relativistic quantum fields was in- vented for and became the robust tool of S-matrix theory of interactions. The present work started from the recognition that Wick's theorem, at least the bosonic one, is the specific case of a simple relationship between operator orderings in general. That is our general Wick theorem. Wick's original contraction is replaced by the general contraction, a form which is fairly straightforward and transparent. A little more involved, yet plausible, is the proposed definition of operator orderings. It covers all common orderings, typical in quantum optics and quantum field theory, and opens a wide perspective toward new ones. The general Wick theorem clarifies the universal structure of orderings. Contrary to naive expectations, the general Wick theorem seems to hold for monomial orderings (permutations) only. Fortunately, we could show that s-orderings of Cahill and Glauber (including the Weyl-Wigner ordering) are reductions of redundant monomial path orderings, hence the general Wick theorem extends for them.

Simple form of the general Wick theorem facilitates analytic calculations. We showed plain explanatory applications in forced quantum mechanical motion, a short derivation of the normal-ordered squeezing operator and a less trivial application to elucidate s-orderings of Cahill and Glauber. The power of general orderings and their Wick theorem would get confirmed further by their future non-trivial utilizations.

It should be admitted on one hand that our proof of the general Wick theorem is tentative. On the other hand, more important is the non-triviality of this proof which tells us about the theorem's depths unless a simpler proof strategy pops up in the future.

The author thanks the Hungarian Scientific Research Fund under Grant No. 124351, and the EU COST Action CA15220 for support. He is also grateful for the anonymous adjudicator of JPA for important criticisms on the previous version of this work.
[1] H. Weyl, Z. Phys. 46, 1 (1927)

[2] E.P. Wigner, Phys. Rev. 40, 749 (1932)

[3] A. Hourlet and A. Kind, Helv. Phys. Acta 22, 319 (1949)

[4] F.J. Dyson, Phys. Rev. 75, 486 (1949)

[5] G.C. Wick, Phys. Rev. 80, 268 (1950)

[6] K.E. Cahill and R.J. Glauber, Phys. Rev. 177, 1857 (1969)

[7] H.W. Lee, Phys. Rep. 259147 (1995)

[8] K. Chou, Zh. Su, B. Hao, and L. Yu, Phys. Rep. 1181 (1985)
[9] J. Campbell, Proc. Lond. Math. Soc. 28, 381 (1897); H. Baker, Proc. Lond. Math. Soc. 34, 347 (1902); F. Hausdorff, Ber. Verh. Saechs. Akad. Wiss. Leipzig 58, 19 (1906)

[10] Fan Hong-Yi Fan and Ruan Tu-Nan, Commun. Theor. Phys. (Beiging) 2, 1289 (1983); Fan Hong-Yi, H.R. Zaidi and J.R. Klauder, Phys. Rev. D35, 1831 (1987); A. Wünsche, J. Optics B: Quantum Semiclass. Opt. 1, R11 (1999). 